\title{
Improved Treatment Efficacy of Antiangiogenic Therapy when Combined with Picornavirus Vaccination in the GL261 Glioma Model
}

\author{
Danielle N. Renner ${ }^{1}$ - Courtney S. Malo ${ }^{2}$ - Fang Jin ${ }^{2} \cdot$ Ian F. Parney ${ }^{2,3}$. \\ Kevin D. Pavelko ${ }^{2}$ • Aaron J. Johnson ${ }^{2,4}$
}

Published online: 30 November 2015

(C) The American Society for Experimental NeuroTherapeutics, Inc. 2015

\begin{abstract}
The addition of antiangiogenic therapy to the standard-of-care treatment regimen for recurring glioblastoma has provided some clinical benefits while also delineating numerous caveats, prompting evaluation of the elicited alterations to the tumor microenvironment. Of critical importance, given the steadily increasing incorporation of immunotherapeutic approaches clinically, is an enhanced understanding of the interplay between angiogenic and immune response pathways within tumors. In the present study, the GL261 glioma mouse model was used to determine the effects of antiangiogenic treatment in an immune-competent host. Following weekly systemic administration of aflibercept, an inhibitor of vascular endothelial growth factor, tumor volume was assessed by magnetic resonance imaging and changes to the tumor microenvironment were determined. Treatment with aflibercept resulted in reduced tumor burden and increased survival compared with controls. Additionally, decreased vascular permeability and preservation of the integrity of tight junction proteins were observed. Treated tumors also displayed hallmarks of anti-angiogenic evasion, including marked upregulation of vascular endothelial growth factor expression and increased tumor invasiveness. Aflibercept
\end{abstract}

Aaron J. Johnson

johnson.aaron2@mayo.edu

1 Neurobiology of Disease Graduate Program, Mayo Clinic, Rochester, MN, USA

2 Department of Immunology, Mayo Clinic, Rochester, MN, USA

3 Department of Neurosurgery, Mayo Clinic, Rochester, MN, USA

4 Department of Neurology, Mayo Clinic, Rochester, MN, USA was then administered in combination with a picornavirusbased antitumor vaccine and tumor progression was evaluated. This combination therapy significantly delayed tumor progression and extended survival beyond that observed for either therapy alone. As such, this work demonstrates the efficacy of combined antiangiogenic and immunotherapy approaches for treating established gliomas and provides a foundation for further evaluation of the effects of antiangiogenic therapy in the context of endogenous or vaccine-induced inflammatory responses.

Key Words Glioma · Combination therapy ·

Antiangiogenic $\cdot$ Tumor invasiveness $\cdot$ Immunotherapy

\section{Introduction}

Glioblastoma multiforme (GBM) is among the most lethal of cancers, in large part because of its high degree of invasiveness and extensive vascularization [1]. While tumor cells initially grow along pre-existing blood vessels, tumor progression is accompanied by an increase in angiogenesis, mediated primarily through the cytokine vascular endothelial growth factor (VEGF) [2,3]. As tumors grow and normal vasculature is disrupted, hypoxic conditions induce endothelial cells and tumor cells to express VEGF and proangiogenic transcription factors, promoting vascular remodeling and sprouting $[2$, 4-6]. Importantly, increased VEGF expression has been shown to correlate with higher tumor grade and decreased patient survival $[7,8]$. These findings have provided rationale for the design of numerous studies aimed at investigating the effects of anti-VEGF therapy on central nervous system (CNS) tumor growth. 
Bevacizumab is a humanized monoclonal antibody that neutralizes circulating VEGF, preventing its interaction with VEGF receptors and subsequent angiogenesis through tumor vessel formation [2,9]. While bevacizumab is the first antiangiogenic therapy approved for the treatment of recurrent GBM, results in clinical trials have been mixed [10]. Phase II clinical trials utilizing bevacizumab for the treatment of recurrent gliomas have demonstrated marked improvements in progression-free survival, although overall survival is only marginally, if at all, affected [11-13]. A more recent phase II trial investigating the effects of bevacizumab on recurrent anaplastic gliomas reported improvement in disease symptoms and increased tumor responsiveness, despite seeing no significant change in progression-free survival or overall survival [14]. Similar results have been reported in newly diagnosed patients receiving bevacizumab concurrently with radiotherapy and temozolomide $[15,16]$. Reports of the effects on patient quality of life, independent living, and performance status have been generally positive, although most agree that the frequency of adverse events following bevacizumab treatment is increased [14, 15, 17, 18].

Similarly, the VEGF inhibitor aflibercept is a recombinant fusion protein consisting of domain 2 of VEGF receptor 1 and domain 3 of VEGF receptor 2 fused to the constant region of human IgG1 [19]. This protein serves as a receptor decoy for both VEGF-A and placental growth factor (PGF). It exhibits higher VEGF binding affinity, improved pharmacokinetic properties, and reduced immunogenicity in humans than previous VEGF receptor decoys [19, 20]. Importantly, unlike other VEGF receptor decoys, aflibercept binds murine VEGF with an affinity similar to that of human VEGF [21-23]. This enhances the value of aflibercept in preclinical testing in a murine model. A preclinical investigation of this drug in mice bearing human gliomas demonstrated moderate efficacy, particularly in animals undergoing prolonged treatment [24]. A phase II clinical trial in which aflibercept was administered to patients with recurrent GBM revealed moderate toxicity but minimal efficacy when used as a monotherapy in the absence of additional treatments [25]. Further studies investigating the value of combining aflibercept with current standard-of-care therapies, as well as novel therapeutic strategies, is warranted.

An important caveat to the use of anti-VEGF therapy for the treatment of GBM is the frequent transition to an increasingly invasive phenotype that occurs following the development of bevacizumab resistance [26, 27]. Incidence of diffuse or distant tumor progression as visualized by $\mathrm{T} 2$-weighted fluid-attenuated inversion recovery (T2-FLAIR) magnetic resonance imaging (MRI) has been reported to be as high as $60 \%$ in some patient cohorts, often in the absence of contrastenhancing lesions [17, 28]. Additionally, a detailed histological analysis of nonenhancing tumor specimens exhibiting FLAIR abnormalities revealed abundant tumor cell infiltration into neuropil [27]. Studies in a variety of animal models using human GBM cell lines have recapitulated these clinical findings. Athymic rats inoculated with G55 cells treated systemically with anti-VEGF antibody demonstrated slowed tumor growth and significantly prolonged survival [29]. However, histological analysis revealed a pattern of increased infiltration, with the formation of numerous satellite tumors [29]. These results were consistently reproduced in orthotopic mouse models, where xenografted human gliomas displayed inhibited tumor growth but significantly increased migration of tumor cells into the brain parenchyma and satellite tumor development [27, 30-32].

While these studies have validated certain clinical findings and provided models using human cells to study phenotypic alterations induced by anti-VEGF treatment, the incorporation of immune-competent models is essential for understanding the complex cellular interactions that occur in the glioma microenvironment. Immune cell infiltration of human GBM is extensive, with monocytes/macrophages representing the largest cellular fraction and growing evidence of $\mathrm{CD} 8+\mathrm{T}$ cell involvement [33, 34]. Both CD8+ and CD4+ T-cell responses have been shown to drive VEGF upregulation in the CNS under neuroinflammatory conditions, which could contribute to angiogenesis and tumor progression [35, 36]. Alternatively, tumor antigen-specific cytotoxic CD8+ T cells and $\mathrm{T}$ helper $1 / 17$ CD4+ T cells have been shown to contribute to antitumor immunity, hindering tumor outgrowth [34, 37-39]. Clinical trials are currently underway combining antiangiogenic and immune-based therapies, including a phase II trial by the Alliance for Clinical Trials in Oncology (clinicaltrials.gov identifier NCT01814813) comparing the efficacy of bevacizumab with and without a heat shock protein-peptide complex (HSPPC-96) vaccine. The availability of preclinical models in which to test such strategies would allow future studies of this nature to be refined to maximize potential efficacy prior to patient enrollment. The GL261 syngeneic glioma in C57BL/6 mice is an immune-competent model commonly used for immunotherapy studies, as inflammation is evident in the brains of GL261-bearing mice [40, 41]. As such, this system is ideally suited for investigating the effects of various treatments, including antiangiogenic therapy, on gliomas in the context of endogenous tumor-elicited neuroinflammatory responses, and the effects of combination therapies, including an immunestimulatory component.

In the present study, GL261 glioma-bearing mice were administered weekly systemic injections of the VEGF inhibitor, aflibercept. Small animal MRI and bioluminescence imaging were employed to examine the effects of treatment on tumor volume. Confocal microscopy was then used to determine alterations in vascular permeability, tight junction integrity, VEGF expression levels, and tumor cell invasiveness. Finally, VEGF inhibition was administered concurrently with an antitumor vaccination therapy to assess the efficacy of combination therapy on tumor progression. 


\section{Methods}

\section{GL261 Tumor Implantation}

The GL261 and GL261-Quad syngeneic glioma cell lines were cultured as previously described $[42,43]$. The GL261Quad cell line is engineered to express the model antigens human gp100 $25-33$, chicken $\mathrm{OVA}_{257-264}$, chicken $\mathrm{OVA}_{323-}$ 339 , and mouse alloantigen $\mathrm{I}_{-E_{52}-68}$. Female 6-8-week-old C57BL/6 mice (The Jackson Laboratory, Bar Harbor, ME, USA) were inoculated by stereotactic injection of $6.0 \times 10^{4}-$ $1.0 \times 10^{5}$ GL261 or GL261-Quad cells into the right striatum. Total injection volume was $1 \mu \mathrm{l}$ administered at a rate of $0.2 \mu \mathrm{l} / \mathrm{min}$. Injection coordinates were $1 \mathrm{~mm}$ lateral, $2 \mathrm{~mm}$ anterior of bregma, at a depth of $3 \mathrm{~mm}$ from the cortical surface. All animal experiments were performed following guidelines set by the Mayo Clinic Institutional Animal Care and Use Committee.

\section{Administration of VEGF Inhibitor and Immunotherapy Vaccine}

Aflibercept (Regeneron Pharmaceuticals, Rensselaer, NY, USA) was administered at a dose of $12.5 \mathrm{mg} / \mathrm{kg}$ by intravenous (i.v.) injection into the tail vein beginning 2 weeks posttumor injection and continuing weekly until mice became moribund and were euthanized. Control mice received an equivalent volume of phosphate buffered saline (PBS) by i.v. injection. The recombinant TMEV Xho1-OVA8 antitumor vaccine was generated as previously described [44]. Two weeks post-tumor injection, mice were administered a single dose of $2 \times 10^{5}$ plaque-forming units of picornavirus by intraperitoneal (i.p.) injection [43].

\section{MRI Acquisition and Analysis}

A Bruker DRX-300 (300 MHz 1H) 7-Tesla vertical-bore small animal imaging system (Bruker Biospin, Billerica, MA, USA) was used to acquire all MR images, similarly to published protocols $[45,46]$. Anesthesia was maintained by inhalation of 3-4\% isofluorane in air, with respiratory rate monitored throughout imaging. Fifteen minutes prior to T1weighted imaging, gadolinium was administered (i.p.) at a dose of $100 \mathrm{mg} / \mathrm{kg}$. The T2-weighted MRI was set as follows: RARE pulse sequence; repetition time $=1500 \mathrm{~ms}$; echo time $=$ $70 \mathrm{~ms}$; RARE factor: 16 ; field of view: $3.2 \times 1.92 \times 1.92 \mathrm{~cm}$; matrix: $256 \times 128 \times 128$. The T1-weighted MRI was as follows: Multi-Slice Multi-Echo (MSME); repetition time: $300 \mathrm{~ms}$; echo time: $9.5 \mathrm{~ms}$; field of view: $4.0 \times 2.0 \times 2.0 \mathrm{~cm}$; matrix: $192 \times 96 \times 96$. Using Analyze 11.0 software (Biomedical Imaging Resource, Mayo Clinic, Rochester, MN, USA) tumor volume was determined by 2 unbiased reviewers by delineating tumor area on consecutive axial slices and generating 3-dimensional volumetric measurements from the acquired dimensions. Tumor volumes as determined by T2weighted MRI performed 2 weeks postinoculation were used for all experiments to divide mice into treatment versus control groups, assuring overall equivalent tumor load in each.

\section{Bioluminescence Imaging}

Bioluminescence imaging was performed using an IVIS Spectrum system (Xenogen Corp., Alameda, CA, USA) running Living Image 4.3 software as previously described [43]. Prior to imaging, animals were administered (i.p.) $150 \mathrm{mg} / \mathrm{kg} \mathrm{D}$ luciferin (Gold Biotehnology, St. Louis, MO, USA). Isofluorane was delivered at $1-2 \%$ via a nose cone system to maintain anesthesia throughout imaging. An exposure time of $10 \mathrm{~s}$ with F-stop=1 was used for acquisition of all images. Signal intensity was quantified as photons/second emitted in a designated region of interest encompassing the head of the animal.

\section{Survival Curve}

Mice were monitored and euthanized when they became moribund, displaying symptoms such as weight loss, hunched posture, and reduced mobility, according to the Mayo Institutional Animal Care and Use Committee standards.

\section{Immunohistochemistry and Vascular Permeability}

One hour prior to brain harvest, mice were injected (i.v.) with $100 \mu 100 \mathrm{mg} / \mathrm{ml}$ fluorescein isothiocyanate (FITC)-bovine serum albumin (Sigma, St. Louis, MO, USA) in PBS. At collection, brains were flash frozen and embedded in Optimal Cutting Temperature compound (Tissue-Tek, Torrance, CA, USA). Tissue sections were prepared as previously described [45]. Briefly, 6- $\mu \mathrm{m}$ sections were fixed in $4 \%$ paraformaldehyde, washed with PBS, and blocked with $5 \%$ normal goat serum containing $0.5 \%$ IGEPAL CA-630 (Sigma) for $1 \mathrm{~h}$. Primary antibodies recognizing claudin-5 (341600, rabbit; Invitrogen, Carlsbad, CA, USA) or VEGF (46154, rabbit; Abcam, Cambridge, UK) were diluted 1:200 in 5\% normal goat serum plus $0.5 \%$ IGEPAL CA-630 and incubated overnight at room temperature. Sections were then washed and secondary antibody goat antirabbit IgG (A-21244; Invitrogen) diluted 1:250 was applied and incubated for $1 \mathrm{~h}$ at room temperature. Sections were counterstained with Hoechst stain diluted 1:500 for $5 \mathrm{~min}$, followed by washing with PBS. After drying, slides were covered with Vectashield medium (Vector Laboratories, Burlingame, CA, USA) and sealed. 


\section{Hematoxylin and Eosin Staining}

Fresh-frozen brains were embedded in Optimal Cutting Temperature compound (Tissue-Tek) and sectioned at $6 \mu \mathrm{m}$. Sections were fixed in $10 \%$ neutral buffered formalin for $10 \mathrm{~min}$, stained with filtered Gill 3X hematoxylin (Thermo, Rockford, IL, USA) for $1 \mathrm{~min}$, differentiated with acid alcohol, and blued with ammonia water for 15 s. Sections were thoroughly washed with water between steps. Counterstaining was achieved via exposure to Eosin-Phloxine (Sigma) for $30 \mathrm{~s}$, followed by dehydration with $95 \%$ alcohol and then absolute alcohol. Slides were rinsed in xylene twice for $5 \mathrm{~min}$ each time, for clearing, and coverslipped with Permount mounting media (Thermo).

\section{Confocal Microscopy}

Images were acquired using an Olympus LEICA DM 25000 confocal microscope equipped with a $40 \times$ or $63 \times$ oil immersion objective and analyzed with LAS AF 6000 acquisition software (Leica Microsystems, Buffalo Grove, IL, USA). Microvessel diameters were measured using the images of claudin-5 tight junction protein staining. For each mouse, 10-20 measurements were obtained and the averaged value for each mouse plotted independently. For analysis of VEGF intensity, images were uploaded into ImageJ (NIH, Bethesda, MD, USA), converted to 8-bit grayscale, and the total intensity of the image area measured.

\section{Statistical Analysis}

Histograms are displayed as mean \pm SEM. Significance between comparisons of 2 groups was determined using Student's $t$ test or Mann-Whitney Rank Sum Test if the data did not follow a normal distribution. Survival differences were determined using the log-rank (Mantel-Cox) test. SigmaStat software (SYSTAT Software Inc., San Jose, CA, USA) was used to perform all calculations.

\section{Results}

\section{GL261 Glioma-bearing Mice Display Reduced Tumor Volume and Increased Survival Following Antiangiogenic Therapy}

To determine the effects of VEGF inhibition on tumor progression in an immune competent murine model, C57BL/6 mice were intracranially inoculated with GL261 syngeneic glioma cells. Two week post-inoculation, animals were scanned using T2-weighted MRI, providing a 3-dimensional rendering and baseline volumetric measurement of each glioma (Fig. 1A, representative animals). With these data, mice were ranked for tumor size and distributed into 2 groups displaying overall equivalent tumor burden. Beginning at 2 weeks and continuing through 5 weeks postinoculation, mice with established tumors received i.v. administration of aflibercept to inhibit systemically the VEGF cytokine, or an equal volume of PBS. At 5 weeks, mice were scanned again and tumor volumes were normalized to the mean value of PBS treated controls (Fig. 1A, B). T2-weighted imaging revealed that mice receiving weekly injections of VEGF inhibitor displayed significantly reduced tumor burden and associated peritumoral edema compared with untreated controls (Fig. 1B). Additionally, bioluminescence imaging was performed weekly on aflibercept treated and control mice, further confirming this reduction in tumor burden by 5 weeks posttumor inoculation (Fig. 1C).

To determine the extent that VEGF inhibition and subsequent reduction in tumor growth results in prolonged survival, treated GL261-bearing mice were allowed to progress until they displayed symptoms of morbidity. Administration of aflibercept or PBS (i.v.) began 2 weeks postinoculation and was maintained weekly until mice became moribund and were euthanized. Median survival of PBS control mice was 46.5 days, while mice receiving weekly aflibercept demonstrated a median survival of 68.0 days (Fig. 1D). As such, inhibition of VEGF significantly prolonged survival of GL261-bearing mice compared with untreated controls.

\section{Inhibition of VEGF Reduces Permeability and Promotes Normalization of Glioma Vasculature}

VEGF inhibition in human GBM results in dramatic reductions in gadolinium-enhancing lesions, suggesting a potential decrease in tumor vascular permeability [14, 47]. Therefore, vascular permeability was assessed in GL261-bearing mice with and without aflibercept treatment. Gliomas were harvested 5 weeks postinoculation, following weekly systemic administration of aflibercept or PBS. One hour prior to harvest, animals were injected (i.v.) with FITC-albumin and subsequent leakage of this high molecular weight compound across the blood-brain barrier was determined. To delineate tumor borders sections were stained with hematoxylin and eosin, and serial sections were analyzed for the level of FITC-albumin leakage from the vasculature. Analysis was performed within the defined tumor area, at the tumor edge, and within normal brain tissue in the contralateral hemisphere for each animal. Mice treated with aflibercept displayed markedly reduced permeability within the tumor area than PBS-treated controls (Fig. 2A). This decrease in FITC-albumin leakage was also noted at the tumor border, although to a lesser extent (Fig. 2A). To further asses the changes in vascular permeability, gadolinium-enhanced T1-weighted MRI was also performed 5 weeks post-tumor inoculation, as tumors are often more permeable to this low molecular weight compound [48]. 
Fig. 1 Antiangiogenic treatment reduces GL261 glioma burden and improves survival. (A) T2weighted magnetic resonance imaging performed 2 weeks postinjection was used to rank tumor volumes to ensure equivalent tumor load in treatment versus control groups. (A, B) T2-weighted magnetic resonance imaging 5 weeks postinjection demonstrates that weekly systemic administration of aflibercept leads to a significant reduction in glioma burden $(n=$ 11 mice/group). (C) Mean bioluminescence intensity (photons/s) demonstrates reduced tumor burden in aflibercept treated animals by 5 weeks posttumor injection $(n=8$ mice/group). (D) GL261-bearing mice receiving weekly intravenous administration of aflibercept ( $n=9$ mice) display prolonged overall survival compared with phosphatebuffered saline-treated controls ( $n=10$ mice). ${ }^{*} p<0.05$; $* * * p<0.001$ a

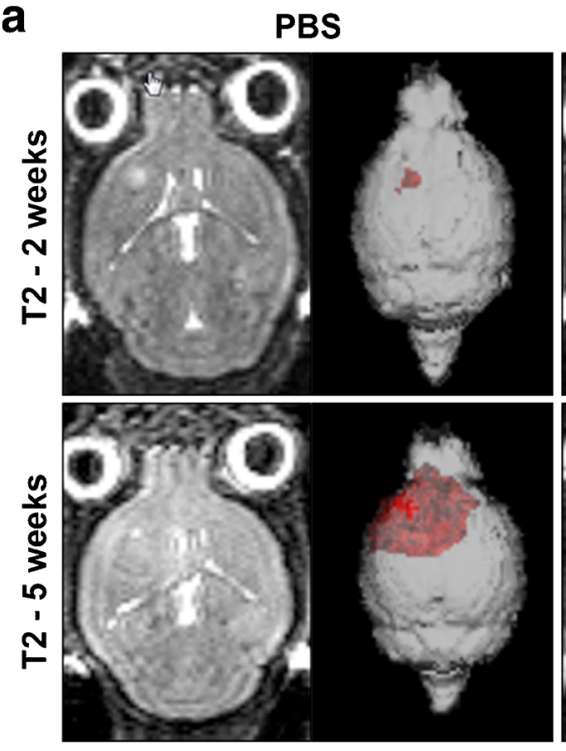

b

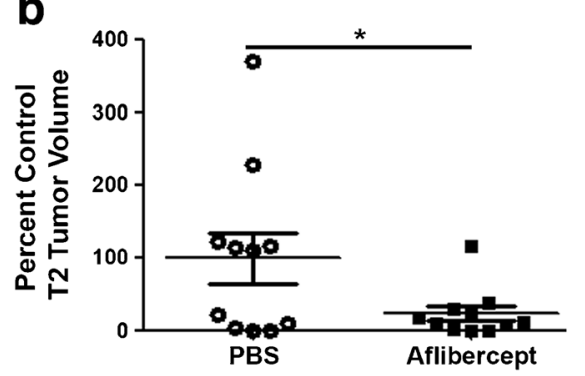

d

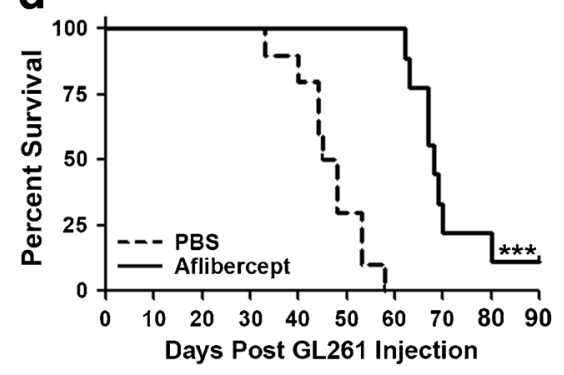

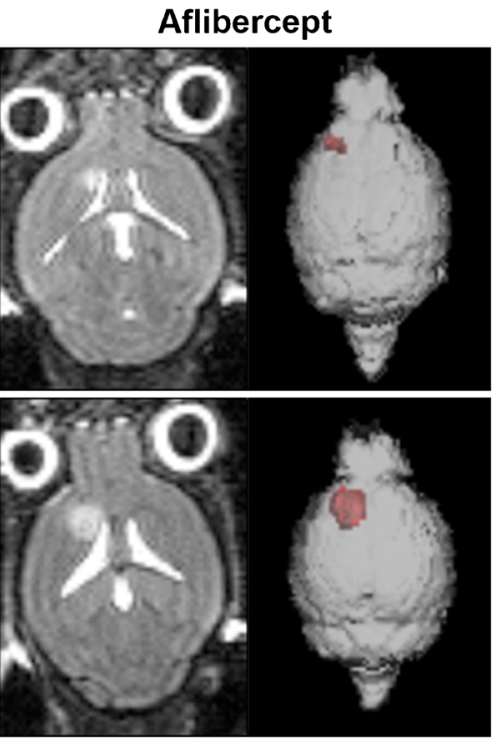

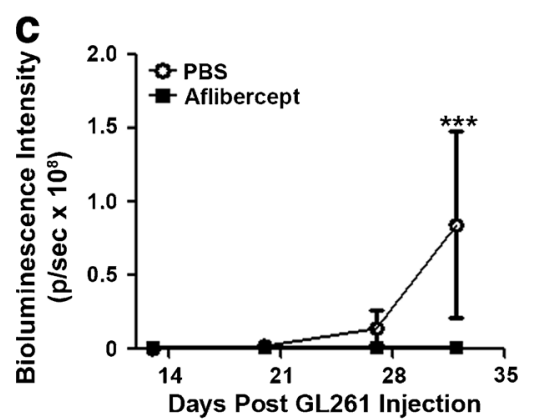

While untreated GL261 gliomas display extensive gadolinium leakage, these scans revealed significantly reduced permeability in aflibercept treated animals compared with PBS controls (Fig. 2B, D). Thus, consistent with human gliomas, treatment with antiangiogenic therapy is shown to reduce tumor vascular permeability in the GL261 glioma model.

In addition to assessing permeability, changes in the size of tumor-associated vasculature were also analyzed. Sections were stained with an antibody recognizing the tight junction protein claudin-5, which outlines microvessels and enables measurement of vessel diameter (Fig. 2A, C). Microvessel diameters from normal brain tissue, within the tumor core, and at the tumor edge were evaluated. In both aflibercept treated and PBS control mice, microvessel diameter was largest within the tumor area. However, VEGF inhibition significantly reduced overall microvessel diameter both within the tumor area and along the tumor edge (Fig. 2A, C). Microvessel diameter within normal brain tissue was not notably different between the treatment groups (Fig. 2A, C). As such, in addition to reducing vascular permeability, blocking VEGF resulted in reduced vasodilation of microvessels and notable vascular normalization in GL261 gliomas.

\section{Antiangiogenic Therapy Results in both Proangiogenic and Proinvasive Evasion Mechanisms in GL261 Gliomas}

While VEGF inhibition in mice bearing GL261 gliomas resulted in decreased tumor burden and increased survival, complete ablation of the tumors was not observed, prompting further analysis of the tumor microenvironment. GL261 gliomas treated weekly with aflibercept or PBS were harvested 5 weeks postinoculation and stained for expression of VEGF cytokine. 
Fig. 2 Aflibercept reduces tumor vascular permeability and normalizes microvessel diameter. (A, B, D) Treatment with aflibercept results in decreased tumor vascular permeability, as determined by fluorescein isothiocyanate (FITC)-albumin leakage from microvessels $(n=$ 4-5 mice/group) and gadoliniumenhanced T1-weighted magnetic resonance imaging $(n=6-7$ mice/group). Volume of gadolinium leakage is measured in voxels ${ }^{3}$, where 1 voxel $=0.21$ mm. (A, C) Control mice $(n=5$ mice) display significantly greater disorganization of tight junction proteins within the tumor area and at the tumor border and larger microvessel diameter than aflibercept-treated mice $(n=4$ mice) at both the tumor core and the tumor edge. Microvessel diameter within the tumor is markedly greater than in normal brain area in both treated and untreated GL261 gliomas. Images were acquired using a $40 \times$ objective. Scale bar $=50 \mu \mathrm{m}$. ${ }^{*} p<0.05 ; * * * p<0.001$. PBS $=$ phosphate-buffered saline

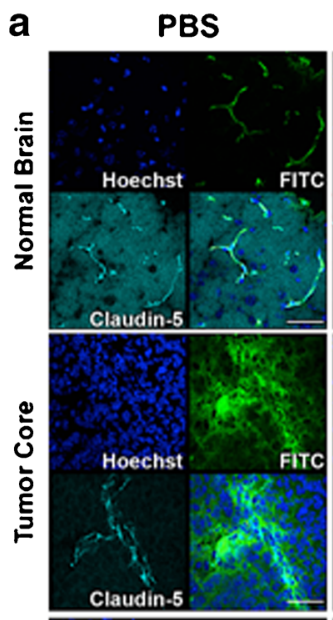

Aflibercept
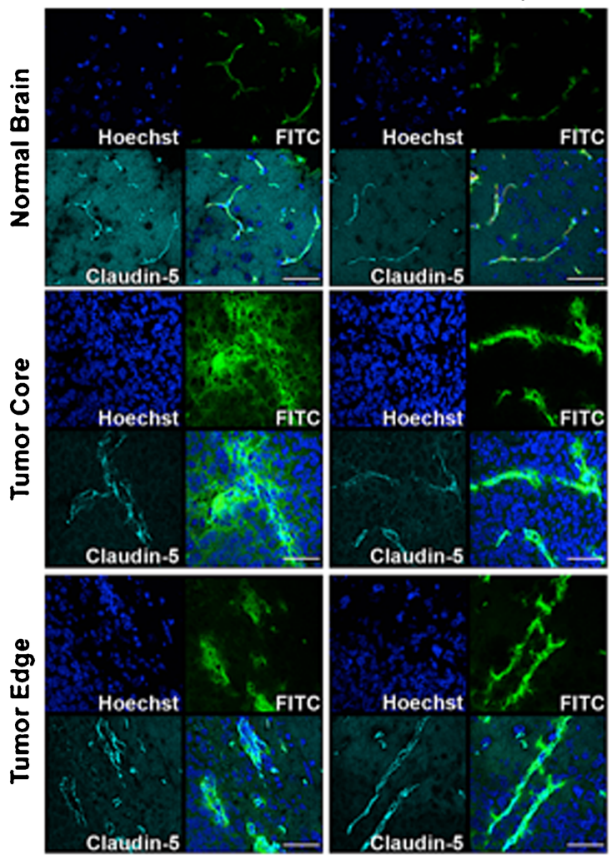

C

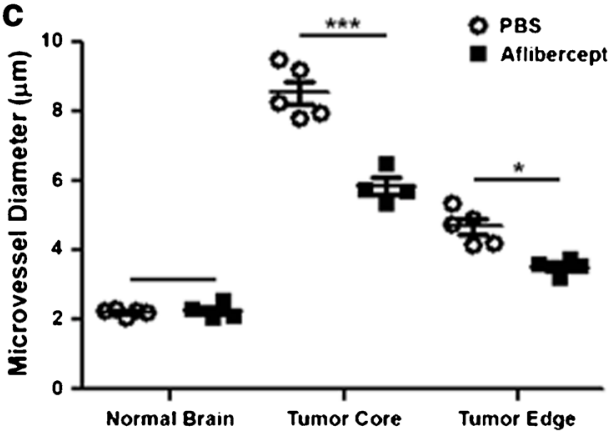

b

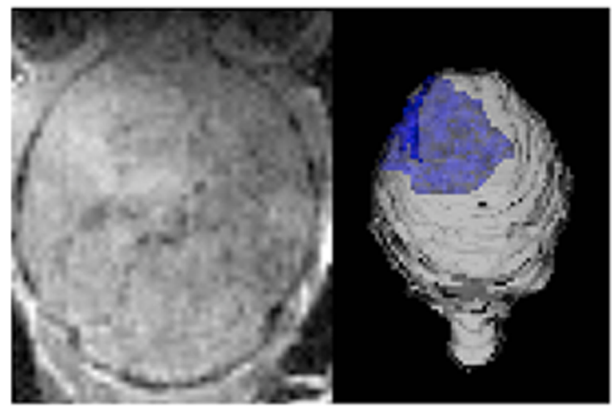

Aflibercept

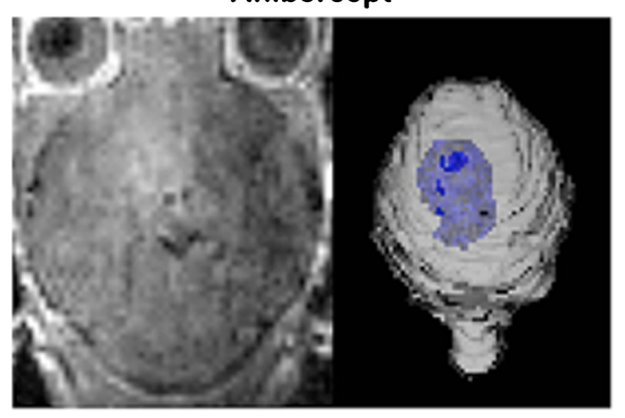

d

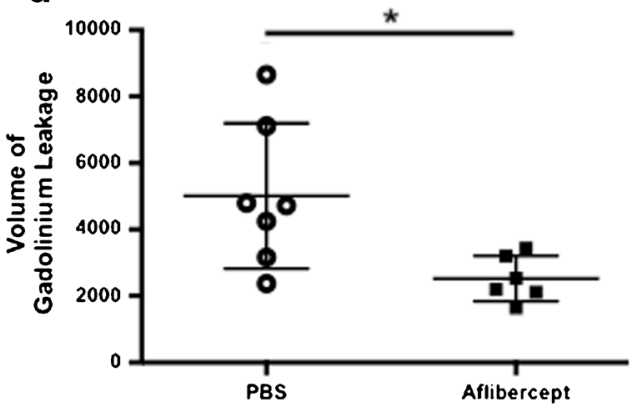

Significantly increased VEGF expression was detected following treatment with aflibercept compared with PBStreated controls (Fig. 3A, B). Therefore, administration of a VEGF inhibitor intended to neutralize VEGF cytokine resulted in increased VEGF production within the glioma environment.

In patients with GBM, VEGF inhibition has been shown to result in an improved quality of life and reduced mortality [11-14, 18, 26]. However, upon recurrence, tumors treated with antiangiogenic therapies display an increasingly invasive phenotype [11, 17, 26-28]. Given this phenotypic alteration observed in human patients, tumor borders following treatment of GL261 gliomas with aflibercept were analyzed. GL261 gliomas receiving weekly administration of aflibercept or PBS were harvested 5 weeks postinoculation and hematoxylin and eosin staining was performed to delineate tumor borders from normal brain tissue, as determined by the dramatic increase in nuclear density within the tumor. PBS-treated tumors exhibited sharp tumor margins and grew as well circumscribed, noninvasive masses (Fig. 4A). Alternatively, aflibercept-treated gliomas displayed significantly more heterogeneous, invasive borders, with tumor cells extending deep into normal brain parenchyma (Fig. 4B). In addition, the formation of satellite tumors was observed following VEGF inhibition (Fig. 4B, arrow). Therefore, despite reducing tumor burden and extending the lifespan of treated mice, antiangiogenic treatment with aflibercept resulted in significantly increased tumor invasiveness.

\section{Combination Antiangiogenic and Immunotherapy Delays Tumor Progression and Improves Survival in GL261 Gliomas}

Recent preclinical and clinical studies have revealed the promise of immunotherapeutic approaches for the treatment of CNS malignancies. Most notably, enhancing tumor infiltrating cytotoxic $\mathrm{T}$ cells has been strongly correlated with improved clinical outcome [34, 49, 50]. Importantly, these novel experimental treatment paradigms are frequently implemented without excluding patients having already undergone current 
a

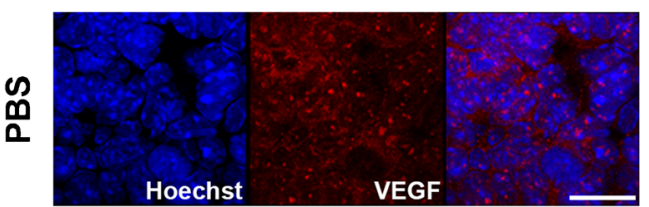

苞

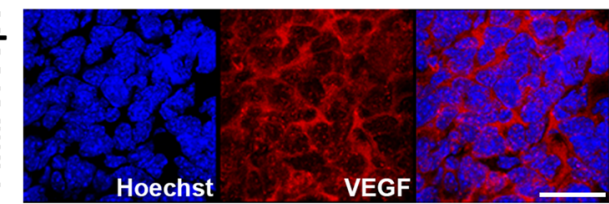

b

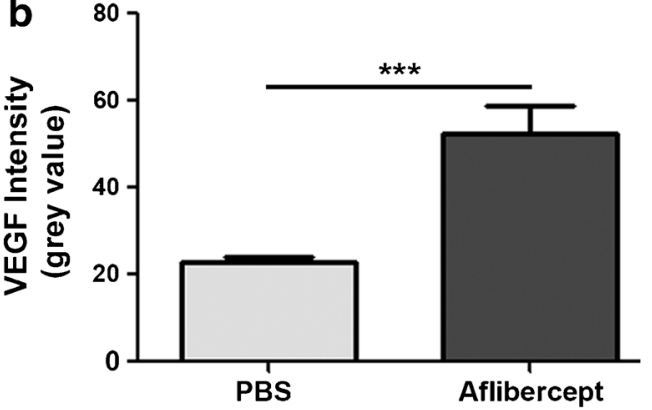

Fig. 3 Vascular endothelial growth factor (VEGF) inhibition results in an upregulation of VEGF expression within gliomas. (A, B) Administration of aflibercept ( $n=12$ mice) results in a significant upregulation of VEGF expression within the tumor environment compared with phosphatebuffered saline (PBS)-treated controls $(n=15$ mice). Images were acquired using a $63 \times$ objective. Scale bar $=40 \mu \mathrm{m} .{ }^{* * *} p<0.001$

standard-of-care therapies, including antiangiogenic therapy $[49,51]$. Additionally, clinical trials assessing the efficacy of combined antiangiogenic and antitumor vaccination approaches are currently being designed and initiated (clinicaltrials.gov identifier NCT01814813). Thus, given the notable interplay between their response pathways, understanding how the concurrent use of antiangiogenic and immune-stimulatory therapies modulates treatment efficacy is of great interest.

To this end, aflibercept was administered alongside a picornavirus-based antitumor vaccine, TMEV Xho1-OVA8, to mice bearing GL261-Quad gliomas (Fig. 5). The GL261Quad glioma is engineered to express model T-cell epitopes, including $\mathrm{OVA}_{257-264}$ [42]. Previous work has established that recombinant TMEV Xho1-OVA8, in which the $\mathrm{OVA}_{257-264}$ epitope is encoded into the leader sequence of the virus, successfully enhances tumor antigen-specific CD8+ T-cell infiltration in the CNS, delaying tumor progression and prolonging survival of glioma-bearing mice [43]. Additionally, an analysis of CD8+ T-cell levels in control or aflibercepttreated GL261-Quad bearing mice revealed a trend toward increased infiltration following VEGF inhibition $(p=0.093$, data not shown), suggesting that antiangiogenic therapy could work synergistically with a cytotoxic T-cell-enhancing vaccine. Two weeks post-GL261-Quad injection, mice were treated with a single dose of TMEV Xho1-OVA8 (i.p.) alone or in a

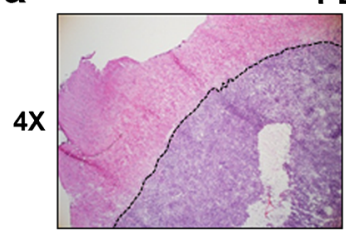

PBS
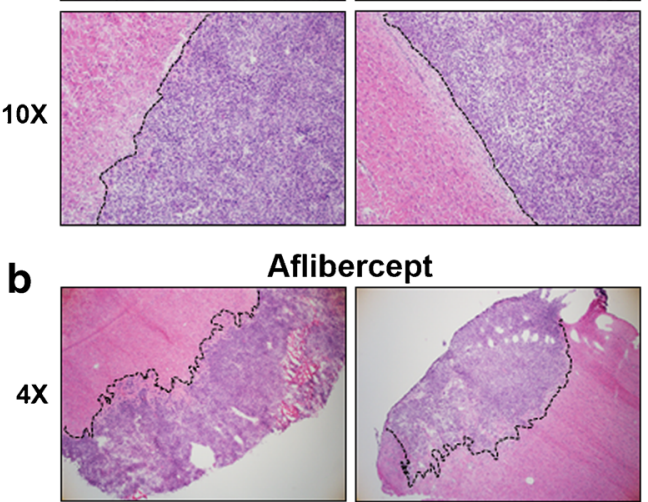

$10 x$
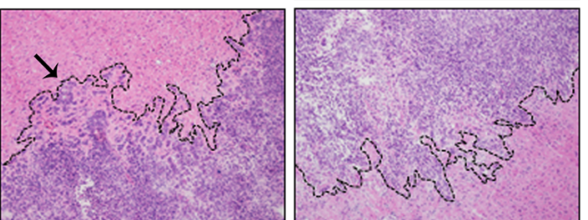

Fig. 4 Antiangiogenic treatment induces increased invasiveness of GL261 gliomas. Representative images of hematoxylin and eosin staining demonstrate that (A) control GL261 gliomas exhibit distinct tumor margins and grow as well-circumscribed, noninvasive masses. (B) Gliomas treated with aflibercept display markedly more invasive borders, with tumor cells migrating into the normal brain parenchyma and satellite tumors arising (arrow). PBS = phosphate-buffered saline

combination with aflibercept (i.v.). Systemic aflibercept administration was maintained weekly in designated treatment groups throughout the course of the experiment until mice displayed symptoms of morbidity. For the vast majority of mice, morbidity was found to correspond to a bioluminescence intensity of approximately $1-2 \times 10^{8}$ photons/s, regardless of the treatment received (Fig. 5A). By 35 days posttumor injection, the combination of aflibercept and TMEV Xho1-OVA8 resulted in significantly reduced tumor burden compared with untreated controls or administration of either treatment alone (Fig. 5A, B). Combined therapy continued to delay tumor progression significantly compared with aflibercept alone to 63 days post-tumor injection (Fig. 5A, B). This delay in tumor progression was accompanied by significantly improved survival, with a median survival of 84 days in mice receiving combined therapy (Fig. 5C). Median survival for PBS controls, picornavirus alone, or aflibercept alone were 39 days, 49 days, and 69 days, respectively (Fig. 5C). Therefore, combined antiangiogenic and immunotherapeutic targeting of gliomas represents a viable treatment approach, as concurrent VEGF inhibition does not preclude cytotoxic T-cell responses against tumor-specific antigens. 
Fig. 5 Combining antiangiogenic and immunotherapies results in enhanced treatment efficacy for GL261 gliomas. (A)

Bioluminescence intensity values [photons/s $(\mathrm{p} / \mathrm{sec})]$ for individual animals, (B) mean bioluminescence intensity (photons/s) per treatment group, and (C) survival curve of GL261Quad glioma-bearing mice administered aflibercept $(n=9$ mice), TMEV Xho1-OVA8 ( $n=9$ mice), or both ( $n=10$ mice) demonstrates a significant delay in tumor progression and increased survival for mice receiving combination therapy compared with phosphatebuffered saline (PBS)-treated controls ( $n=9$ mice) or either treatment alone. (B) Asterisks denote significance as determined at 35 days post-tumor injection. Differences in tumor burden of aflibercept versus combined treatment groups remains significant through 63 days postinjection $(p=0.009)$. ${ }^{*} p<0.05 ;{ }^{* *} p<0.01$; $* * * p<0.001$ a
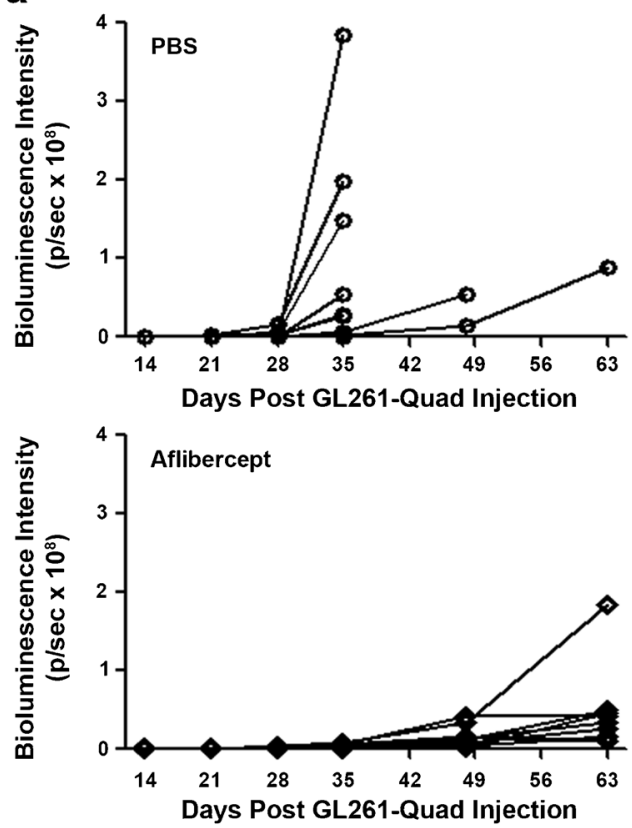

b

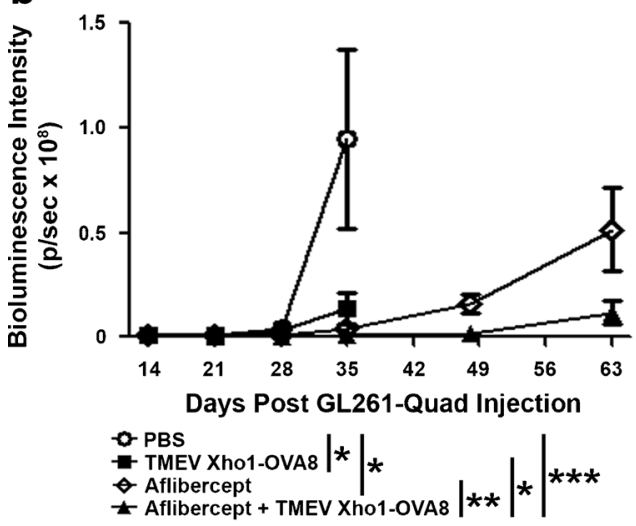

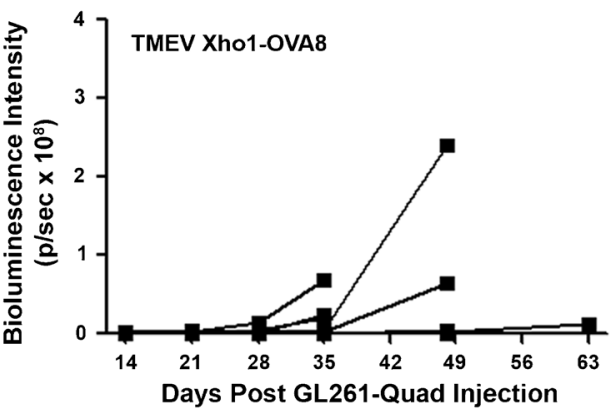

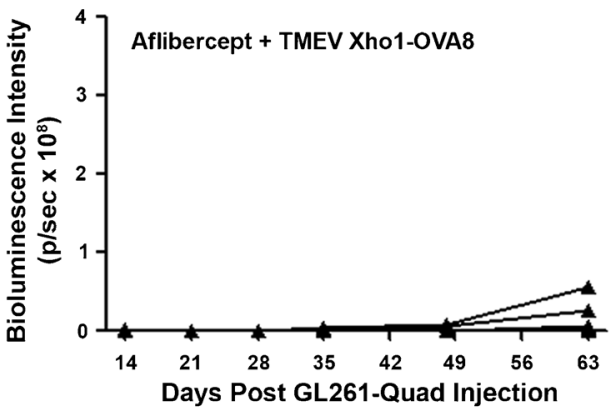

C

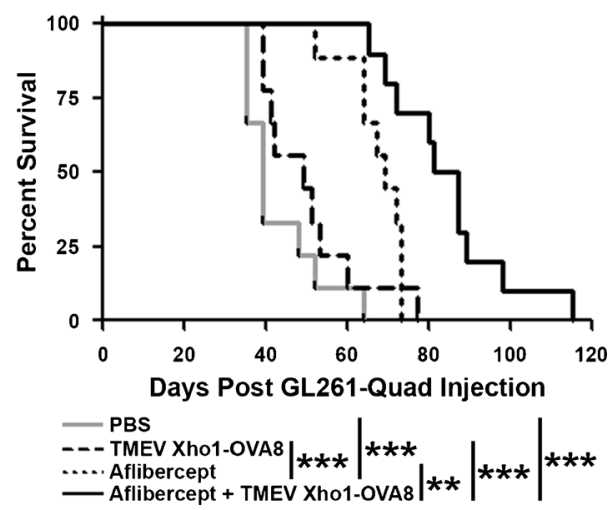

\section{Discussion}

The current study utilizes the immune-competent GL261 murine glioma model to investigate the influence of VEGF inhibition on tumor progression and to evaluate the efficacy of combination antiangiogenic and immunotherapeutic treatment approaches. The findings herein confirm the utility of incorporating the GL261 model system into studies investigating the complex interactions between angiogenic and immune response pathways, as the responses in this model recapitulate several features observed in human GBM following VEGF-targeted therapy. Tumor burden was reduced and survival prolonged; however, tumors became more invasive, with VEGF expression upregulated in the tumor milieu and glioma cell infiltration dramatically increased. Additionally, this work demonstrates that antiangiogenic therapy can successfully be employed in conjunction with a cytotoxic T-cell-enhancing antitumor vaccine to improve treatment efficacy. Clinical trials combining antiangiogenic and immune-based therapies for the treatment of recurrent GBM are already being carried out (clinicaltrials.gov identifier NCT01814813) and the availability of relevant preclinical models will provide valuable data that for optimizing the designs of such studies.

MRI is the primary imaging modality employed clinically to assess progression of human GBM. GBM typically presents with both gadolinium-enhancement, as visible by T1weighted MRI with contrast, and significant peritumoral edema, as seen by T2-weighted imaging [52-54]. Tumor response and relapse have historically been detected following the same criteria. However, several recent studies have noted an atypical pattern of progression in some patients treated with antiangiogenic therapy, with an absence of contrast-enhancing lesions despite continued T2-FLAIR signal [27]. Preclinical studies using bevacizumab to treat human tumor xenografts have confirmed this effect, demonstrating reductions in vascular leakage as visualized by MRI, with tumors restored to reduced degree of permeability to small molecular weight tracers $[55,56]$. Importantly, GL261 gliomas similarly display 
dramatically reduced vascular permeability following VEGF inhibition, as evidenced by increased integrity of tight junction proteins and decreased FITC-albumin and gadolinium leakage into the tumor. To explain the observed differences in presentation of recurrent gliomas, Lu and Bergers [17] have proposed 2 primary mechanisms by which these tumors evade the effects of antiangiogenic therapy: proangiogenic and proinvasive. Proangiogenic evasion involves the upregulation of compensatory angiogenic factors to restore neovascularization following the loss of VEGF signaling. Alternatively, proinvasive evasion results in altered gene expression patterns that promote a migratory phenotype, allowing tumor cells to co-opt existing vasculature outside of the initial tumor site. Interestingly, GL261 gliomas appear to provide a model that incorporates both of these evasive mechanisms. These tumors display upregulation of VEGF expression within the tumor environment while also shifting to an increasingly invasive phenotype. Understanding the interplay between altered signaling pathways that contribute to each of these evasive mechanisms in CNS tumors following antiangiogenic therapy could provide insight into processes governing disparate response patterns observed clinically.

Upregulation of VEGF expression within the tumor microenvironment following antiangiogenic therapy prompts future work aimed at delineating the mechanism(s) underlying this increase in expression. The effects of VEGF in gliomas have been shown to be enhanced by hypoxia, tumor cell expression of the cytokine, and hematopoietic derived factors $[4,5,57]$. More recently, CD8+ and CD4+ T cells have been demonstrated to promote VEGF upregulation in the CNS under neuroinflammatory conditions $[35,36]$. While enhanced VEGF expression in our model is apparent, the source of this increase requires further investigation. In addition, potential upregulation of related proangiogenic factors, such as tumor necrosis factor- $\alpha$, interleukin- 1 family members, basic fibroblast growth factor, and stromal cell-derived factor- $1 \alpha$, remains to be determined [17]. Importantly, a number of these signaling molecules play a significant role in a variety of immune response pathways as well, underlining the importance of the immune-competent GL261 model in future work aimed at delineating the respective contributions of the involved systems.

The increased tumor invasiveness that results following antiangiogenic therapy creates an increasingly complex tumor landscape upon which novel therapeutic approaches can be applied. The near certainty of recurrence following the standard treatment regimen of surgical resection and chemotherapy and radiation underscores the inherent difficulty in identifying and removing all of the malignant cells present. Augmenting the migratory capabilities of these cells by administering VEGF inhibitors in recurrent GBM cases further confounds this issue. As such, elimination of these cells will require the application of highly precise and selective targeting mechanisms. The immune system is optimally designed for such an application, and effective immunotherapy remains a promising approach for eradicating tumor cells that persist following current standard-of-care therapies. However, understanding how antiangiogenic and immune-based therapies interact to modulate treatment efficacy is essential for successful implementation of such combined treatment strategies clinically.

The present study demonstrates that concurrent VEGF inhibition alongside an antitumor vaccine to enhance cytotoxic T cells more effectively treats GL261 gliomas than either treatment alone. Future work will be aimed at understanding the mechanisms underlying this augmented response, including any synergistic effects of combined therapy. Previous studies in nonCNS solid tumors have shown the ability of VEGF inhibition to enhance $\mathrm{CD} 8+\mathrm{T}$-cell responses $[58,59]$. While not reaching statistical significance, our data also revealed a trend toward increased CD8+ T-cell infiltration into the CNS following treatment with aflibercept, suggesting that vascular normalization could, in fact, promote immune cell infiltration. Importantly, the dose of VEGF inhibitor has been shown to modulate the level of immune cell infiltration, and may require further optimization for enhancing immunotherapeutic responses in our model [60]. Additionally, detailed characterizations of the effector functions of infiltrating immune cells, including alterations to cytotoxic function and immunosuppressive capacity, are underway. Furthermore, reciprocal studies will investigate altered angiogenic responses following enhancement of antitumor responses, as immune cells have also been shown to modulate VEGF expression and angiogenesis [35, 36].

The findings of the current study demonstrate that GL261 gliomas recapitulate several features of human GBM following administration of antiangiogenic therapy. In these tumors, VEGF inhibition results in decreased tumor burden, extended survival, and reduced vascular permeability. However, tumors evade complete eradication and develop markedly more aggressive characteristics, including upregulated VEGF expression and increased invasiveness. Therefore, this system provides a unique model for future work aimed at understanding the interplay between the evasive mechanisms employed by gliomas following antiangiogenic therapy in the context of an endogenous antitumor immune response. Additionally, this study demonstrates the efficacy of combining antiangiogenic and immune-modulatory therapies for improved treatment of established gliomas and establishes the GL261 model as an effective system in which to study the complex interactions between angiogenic and immune response pathways. 
Acknowledgments We thank Dr. Raymond Iezzi for providing reagents used in this study. We thank the Mayo Clinic Nuclear Magnetic Resonance core facility, especially Dr. Slobodan I. Macura and Dr. Prasanna K. Mishra, for technical assistance with the 7-Tesla small animal imaging. This work was supported by a grant from the Brains Together for a Cure organization, a Minnesota Partnership for Biotechnology and Medical Genomics Grant (\#89) and the National Institutes of Health [R21 CA186976; F31 CA180299].

\section{Compliance with Ethical Standards}

Required Author Forms Disclosure forms provided by the authors are available with the online version of this article.

\section{References}

1. Jain RK, di Tomaso E, Duda DG, Loeffler JS, Sorensen AG, Batchelor TT. Angiogenesis in brain tumours. Nat Rev Neurosci 2007;8:610-622.

2. Soda Y, Myskiw C, Rommel A, Verma IM. Mechanisms of neovascularization and resistance to anti-angiogenic therapies in glioblastoma multiforme. J Mol Med (Berl) 2013;91:439-448.

3. Holash J, Maisonpierre PC, Compton D, et al. Vessel cooption, regression, and growth in tumors mediated by angiopoietins and VEGF. Science 1999;284:1994-1998.

4. Shweiki D, Itin A, Soffer D, Keshet E. Vascular endothelial growth factor induced by hypoxia may mediate hypoxia-initiated angiogenesis. Nature 1992;359:843-845.

5. Plate KH, Breier G, Weich HA, Risau W. Vascular endothelial growth factor is a potential tumour angiogenesis factor in human gliomas in vivo. Nature 1992;359:845-848.

6. Kim KJ, Li B, Winer J, et al. Inhibition of vascular endothelial growth factor-induced angiogenesis suppresses tumour growth in vivo. Nature 1993;362:841-844.

7. Chaudhry IH, O'Donovan DG, Brenchley PE, Reid H, Roberts IS. Vascular endothelial growth factor expression correlates with tumour grade and vascularity in gliomas. Histopathology 2001;39: 409-415.

8. Oehring RD, Miletic M, Valter MM, et al. Vascular endothelial growth factor (VEGF) in astrocytic gliomas-a prognostic factor? J Neurooncol 1999;45:117-125.

9. Presta LG, Chen H, O'Connor SJ, et al. Humanization of an antivascular endothelial growth factor monoclonal antibody for the therapy of solid tumors and other disorders. Cancer Res 1997;57: 4593-4599.

10. Cohen MH, Shen YL, Keegan P, Pazdur R. FDA drug approval summary: bevacizumab (Avastin) as treatment of recurrent glioblastoma multiforme. Oncologist 2009;14:1131-1138.

11. Norden AD, Drappatz J, Muzikansky A, et al. An exploratory survival analysis of anti-angiogenic therapy for recurrent malignant glioma. J Neurooncol 2009;92:149-155.

12. Friedman HS, Prados MD, Wen PY, et al. Bevacizumab alone and in combination with irinotecan in recurrent glioblastoma. J Clin Oncol 2009;27:4733-4740.

13. Vredenburgh JJ, Desjardins A, Herndon JE, 2nd, et al. Bevacizumab plus irinotecan in recurrent glioblastoma multiforme. J Clin Oncol 2007;25:4722-4729.

14. Kreisl TN, Zhang W, Odia Y, et al. A phase II trial of single-agent bevacizumab in patients with recurrent anaplastic glioma. Neuro Oncol 2011;13:1143-1150.
15. Chinot OL, Wick W, Mason W, et al. Bevacizumab plus radiotherapy-temozolomide for newly diagnosed glioblastoma. $\mathrm{N}$ Engl J Med 2014;370:709-722.

16. Gilbert MR, Dignam JJ, Armstrong TS, et al. A randomized trial of bevacizumab for newly diagnosed glioblastoma. N Engl J Med 2014;370:699-708.

17. Lu KV, Bergers G. Mechanisms of evasive resistance to anti-VEGF therapy in glioblastoma. CNS Oncol 2013;2:49-65.

18. Nagpal S, Harsh G, Recht L. Bevacizumab improves quality of life in patients with recurrent glioblastoma. Chemother Res Pract 2011;2011:602812.

19. Holash J, Davis S, Papadopoulos N, et al. VEGF-Trap: a VEGF blocker with potent antitumor effects. Proc Natl Acad Sci U S A 2002;99:11393-11398.

20. Lockhart AC, Rothenberg ML, Dupont J, et al. Phase I study of intravenous vascular endothelial growth factor trap, aflibercept, in patients with advanced solid tumors. J Clin Oncol 2010;28:207214.

21. Papadopoulos N, Martin J, Ruan Q, et al. Binding and neutralization of vascular endothelial growth factor (VEGF) and related ligands by VEGF Trap, ranibizumab and bevacizumab. Angiogenesis 2012;15:171-185.

22. Yu L, Wu X, Cheng Z, et al. Interaction between bevacizumab and murine VEGF-A: a reassessment. Invest Ophthalmol Visual Sci 2008;49:522-527.

23. Ferrara N, Hillan KJ, Gerber H-P, Novotny W. Discovery and development of bevacizumab, an anti-VEGF antibody for treating cancer. Nat Rev Drug Discov 2004;3:391-400.

24. Gomez-Manzano C, Holash J, Fueyo J, et al. VEGF Trap induces antiglioma effect at different stages of disease. Neuro Oncol 2008;10:940-945.

25. de Groot JF, Lamborn KR, Chang SM, et al. Phase II study of aflibercept in recurrent malignant glioma: a North American Brain Tumor Consortium study. J Clin Oncol 2011;29:2689-2695.

26. Narayana A, Gruber D, Kunnakkat S, et al. A clinical trial of bevacizumab, temozolomide, and radiation for newly diagnosed glioblastoma. J Neurosurg 2012;116:341-345.

27. de Groot JF, Fuller G, Kumar AJ, et al. Tumor invasion after treatment of glioblastoma with bevacizumab: radiographic and pathologic correlation in humans and mice. Neuro Oncol 2010;12:233242.

28. Zuniga RM, Torcuator R, Jain R, et al. Efficacy, safety and patterns of response and recurrence in patients with recurrent high-grade gliomas treated with bevacizumab plus irinotecan. J Neurooncol 2009;91:329-336.

29. Rubenstein JL, Kim J, Ozawa T, et al. Anti-VEGF antibody treatment of glioblastoma prolongs survival but results in increased vascular cooption. Neoplasia 2000;2:306-314.

30. Lamszus K, Kunkel P, Westphal M. Invasion as limitation to antiangiogenic glioma therapy. Acta Neurochir Suppl 2003;88:169177.

31. Keunen O, Johansson M, Oudin A, et al. Anti-VEGF treatment reduces blood supply and increases tumor cell invasion in glioblastoma. Proc Natl Acad Sci U S A 2011;108:3749-3754.

32. Paez-Ribes M, Allen E, Hudock J, et al. Antiangiogenic therapy elicits malignant progression of tumors to increased local invasion and distant metastasis. Cancer Cell 2009;15:220-231.

33. Parney IF, Waldron JS, Parsa AT. Flow cytometry and in vitro analysis of human glioma-associated macrophages. Laboratory investigation. J Neurosurg 2009;110:572-582.

34. Barcia C, Jr., Gomez A, Gallego-Sanchez JM, et al. Infiltrating CTLs in human glioblastoma establish immunological synapses with tumorigenic cells. Am J Pathol 2009;175:786-798.

35. Suidan GL, Dickerson JW, Chen Y, et al. CD8 T cell-initiated vascular endothelial growth factor expression promotes central nervous 
system vascular permeability under neuroinflammatory conditions. J Immunol 2010;184:1031-1040.

36. Argaw AT, Asp L, Zhang J, et al. Astrocyte-derived VEGF-A drives blood-brain barrier disruption in CNS inflammatory disease. J Clin Invest 2012;122:2454-2468.

37. Ueda R, Fujita M, Zhu X, et al. Systemic inhibition of transforming growth factor-beta in glioma-bearing mice improves the therapeutic efficacy of glioma-associated antigen peptide vaccines. Clin Cancer Res 2009;15:6551-6559.

38. Xiong Z, Ohlfest JR. Topical imiquimod has therapeutic and immunomodulatory effects against intracranial tumors. J Immunother 2011;34:264-269.

39. Cantini G, Pisati F, Mastropietro A, et al. A critical role for regulatory $\mathrm{T}$ cells in driving cytokine profiles of Th17 cells and their modulation of glioma microenvironment. Cancer Immunol Immunother 2011;60:1739-1750.

40. Ausman JI, Shapiro WR, Rall DP. Studies on the chemotherapy of experimental brain tumors: development of an experimental model. Cancer Res 1970;30:2394-2400.

41. Maes W, Van Gool SW. Experimental immunotherapy for malignant glioma: lessons from two decades of research in the GL261 model. Cancer Immunol Immunother 2011;60:153-160.

42. Ohlfest JR, Andersen BM, Litterman AJ, et al. Vaccine injection site matters: qualitative and quantitative defects in CD8 T cells primed as a function of proximity to the tumor in a murine glioma model. J Immunol 2013;190:613-620.

43. Renner DN, Jin F, Litterman AJ, et al. Effective treatment of established GL261 murine gliomas through picornavirus vaccination-enhanced tumor antigen-specific CD8+ T cell responses. PLoS One 2015;10:e0125565.

44. Pavelko KD, Bell MP, Karyampudi L, et al. The epitope integration site for vaccine antigens determines virus control while maintaining efficacy in an engineered cancer vaccine. Mol Ther 2013;21:10871095.

45. Johnson HL, Chen Y, Jin F, et al. CD8 T cell-initiated blood-brain barrier disruption is independent of neutrophil support. J Immunol 2012;189:1937-1945.

46. Pirko I, Johnson AJ. Neuroimaging of demyelination and remyelination models. Curr Top Microbiol Immunol 2008;318: 241-266.

47. $\mathrm{Xu} \mathrm{T}$, Chen J, Lu Y, Wolff JE. Effects of bevacizumab plus irinotecan on response and survival in patients with recurrent malignant glioma: a systematic review and survival-gain analysis. BMC Cancer 2010;10:252.

48. Lockman PR, Mittapalli RK, Taskar KS, et al. Heterogeneous blood-tumor barrier permeability determines drug efficacy in experimental brain metastases of breast cancer. Clin Cancer Res 2010;16:5664-5678.

49. Prins RM, Soto H, Konkankit V, et al. Gene expression profile correlates with T-cell infiltration and relative survival in glioblastoma patients vaccinated with dendritic cell immunotherapy. Clin Cancer Res 2011;17:1603-1615.

50. Wheeler CJ, Black KL, Liu G, et al. Vaccination elicits correlated immune and clinical responses in glioblastoma multiforme patients. Cancer Res 2008;68:5955-5964.

51. Schuessler A, Smith C, Beagley L, et al. Autologous T-cell therapy for cytomegalovirus as a consolidative treatment for recurrent glioblastoma. Cancer Res 2014;74:3466-3476.

52. Iwama T, Yamada H, Sakai N, et al. Correlation between magnetic resonance imaging and histopathology of intracranial glioma. Neurol Res 1991;13:48-54.

53. Chen XZ, Yin XM, Ai L, Chen Q, Li SW, Dai JP. Differentiation between brain glioblastoma multiforme and solitary metastasis: qualitative and quantitative analysis based on routine MR imaging. AJNR Am J Neuroradiol 2012;33;1907-1912.

54. Hammoud MA, Sawaya R, Shi W, Thall PF, Leeds NE. Prognostic significance of preoperative MRI scans in glioblastoma multiforme. J Neurooncol 1996;27;65-73.

55. Pishko GL, Muldoon LL, Pagel MA, Schwartz DL, Neuwelt EA. Vascular endothelial growth factor blockade alters magnetic resonance imaging biomarkers of vascular function and decreases barrier permeability in a rat model of lung cancer brain metastasis. Fluids Barriers CNS 2015;12:5.

56. Muldoon LL, Gahramanov S, Li X, Marshall DJ, Kraemer DF, Neuwelt EA. Dynamic magnetic resonance imaging assessment of vascular targeting agent effects in rat intracerebral tumor models. Neuro Oncol 2011;13:51-60.

57. Du R, Lu KV, Petritsch C, et al. HIF1alpha induces the recruitment of bone marrow-derived vascular modulatory cells to regulate tumor angiogenesis and invasion. Cancer Cell 2008;13:206-220.

58. Hodi FS, Lawrence D, Lezcano C, et al. Bevacizumab plus ipilimumab in patients with metastatic melanoma. Cancer Immunol Res 2014;2:632-642.

59. Mansfield AS, Nevala WK, Lieser EA, Leontovich AA, Markovic $\mathrm{SN}$. The immunomodulatory effects of bevacizumab on systemic immunity in patients with metastatic melanoma. Oncoimmunology 2013;2:e24436.

60. Huang Y, Yuan J, Righi E, et al. Vascular normalizing doses of antiangiogenic treatment reprogram the immunosuppressive tumor microenvironment and enhance immunotherapy. Proc Natl Acad Sci U S A 2012;109:17561-17566. 